\title{
Colonic duplication in an adult who presented with chronic constipation attributed to hypothyroidism
}

\author{
Tihomir Kekez, Goran Augustin, Irena Hrstic, Dubravko Smud, Mate Majerovic, Zeljko Jelincic, Emil Kinda
}

Tihomir Kekez, Goran Augustin, Dubravko Smud, Zeljko Jelincic, Mate Majerovic, Emil Kinda, Department of Surgery, Division of Abdominal Surgery, Clinical Hospital Center Zagreb, Kispaticeva 12, 10000 Zagreb, Croatia

Irena Hrstic, Department of Internal medicine, Division of Gastroenterology, Clinical Hospital Center Zagreb, Kispaticeva 12, 10000 Zagreb, Croatia

Correspondence to: Goran Augustin, Department of Surgery, Division of Abdominal Surgery, Clinical Hospital Center Zagreb, Stupnička 6, 10000 Zagreb, Croatia. augustin.goran@gmail.com Telephone: +385-91-5252372 Fax: +385-1-2421845

Received: October 24, 2007 Revised: December 5, 2007

\begin{abstract}
Gastrointestinal duplications are an uncommon congenital abnormality that manifest before the age of two in $80 \%$ of cases. Ileal duplication is the most common while colonic duplication, either cystic or tubular, occurs in 10\%-15\% of cases and remains asymptomatic and undiagnosed in most cases. Mostly occurring in pediatric patients, colonic duplication is encountered in adults in only a few cases. The most common clinical manifestations are abdominal pain and intestinal obstruction. Rarely, duplications present with signs of acute abdomen or acute bleeding. This study reports a case of colonic duplication in an adult who presented with chronic constipation. Complete diagnostic workup was made on several occasions during the previous eight year period, but no pathology was found and chronic constipation was attributed to hypothyroidism caused by long standing Hashimoto thyroiditis. Multislice CT, performed because of abdominal distension, defined colonic pathology but the definite diagnosis of duplication of the transversal colon was made at operation. The cystic duplication and the adjacent part of the ascending and transversal colon were excised enblock. This study implies that colonic duplication, though uncommon, should be included in the differential diagnosis of chronic constipation even when precipitating factors for constipation, such as hypothyroidism are present.
\end{abstract}

(C) 2008 WJG. All rights reserved.

Key words: Colonic duplication; Constipation; Abdominal distension; Hypothyroidism

Peer revriewer: Robin G Lorenz, Associate Professor, Department of Pathology, University of Alabama at Birmingham, 845 19th Street South BBRB 730, Birmingham, AL 35294-2170, United States
Kekez T, Augustin G, Hrstic I, Smud D, Majerovic M, Jelincic Z, Kinda E. Colonic duplication in an adult who presented with chronic constipation attributed to hypothyroidism. World $J$ Gastroenterol 2008; 14(4): 644-646 Available from: URL: http:// www.wjgnet.com/1007-9327/14/644.asp DOI: http://dx.doi. org/10.3748/wjg.14.644

\section{INTRODUCTION}

Duplications are rare congenital cystic or tubular abnormalities of the gastrointestinal tract that occur anywhere from the mouth to the anus. Despite a variety of early descriptions, Ladd coined the term alimentary tract duplications in 1937, which described the clinical and pathologic aspects of these lesions more effectively ${ }^{[1]}$. Their etiology is of embryonic origin and several theories have been proposed. However, none of these theories alone is able to explain the full diversity of these lesions. Although they can present at any age, more than $80 \%$ of the cases present before the age of two as an acute abdomen or bowel obstruction ${ }^{[2]}$. Colorectal duplications occur in $13 \%$ of these cases ${ }^{[3]}$. If encountered incidentally, these lesions should be surgically addressed to avoid any future complications ${ }^{[4,5]}$.

We report a case of colonic duplication in an adult woman who presented with distended abdomen and chronic constipation that was attributed to long standing hypothyroidism.

\section{CASE REPORT}

A 42-year-old woman was admitted to the division of gastroenterology with symptoms of significant abdominal distension and chronic constipation. The patient had a medical history of chronic constipation from the early childhood which was not striking until 1991 when, during gestation, significant gaseous bowel distention was found. Complete diagnostic workup was made to find the cause of the constipation but all findings were normal. The constipation was attributed to hypothyroidism caused by long standing Hashimoto thyroiditis treated with thyroid hormone replacement. In 1999 the symptoms progressed and again complete diagnostic workup was made. The diagnosis was elongated colon (dolichocolon). During these eight years the patient used cathartics, different types of tea and manual stool evacuation. There were periods of seven days without stool evacuation. The patient got 


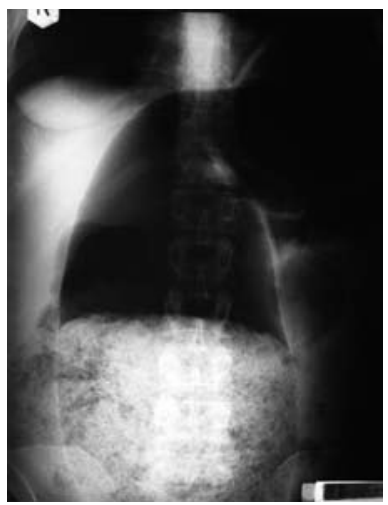

Figure 1 Plain X-ray of the abdomen showing a dilated intestinal loop occupying more than half of the abdominal cavity filled with fecal masses.

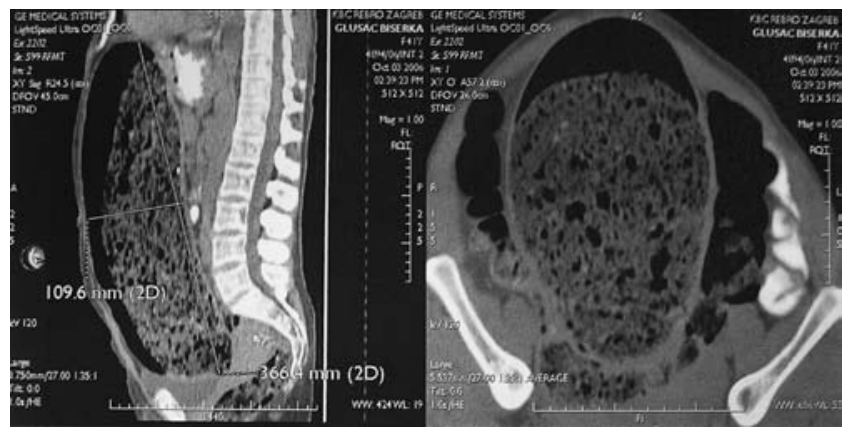

Figure 2 Abdominal CT showing a dilated intestine from the diaphargm to the pelvis, measuring $36 \mathrm{~cm}$ in length with a diameter of $11 \mathrm{~cm}$ dislocating normal intestine laterally.

used to irregular bowel habits and after 1999 did not go to regular controls. Finally in the last few months in 2006 she noticed significant abdominal distension and consulted the gastroenterologist. Complete laboratory findings, including proteins, albumins, IgA, IgM, IgG, T3, T4 and TSH were normal. ECG and esophagogastroduodenoscopy were normal. Plain X-rays of the abdomen showed an extremely dilated intestinal loop, presumably colon with fecal masses without signs of obstruction (Figure 1). Colonoscopy revealed normal mucosa with few biopsies showing chronic nonspecific colitis. Anal manometry showed: lower mean resting pressure $(50 \mathrm{mmHg})$, normal squeeze pressure (200 $\mathrm{mmHg}$ ), normal rectal sensation, rectal compliance and recto-anal inhibitory reflex. The conclusion was that there were no elements for Hirschsprung's disease and that the internal anal sphincter showed slight hypofunction. Multislice CT with peroral contrast showed an enormously dilated intestinal loop from diaphragm to pelvis, measuring $36 \mathrm{~cm}$ in length with the diameter of $11 \mathrm{~cm}$ occupying the complete abdominal cavity in the midline (Figure 2). The stomach, rectum and genitourinary tract were normal. There were no enlarged lymph nodes. The patient was transferred to the department of surgery and the exploratory laparotomy revealed colonic duplication of the transversal colon with no other intraabdominal pathology (Figure 3). Duplication was separated by a sharp dissection from the left half of the transversal colon but shared common wall with the right half of the transversal colon. The duplication ended blindly at the splenic flexure and had an opening that communicated with the normal colon at the hepatic flexure (Figure 4).

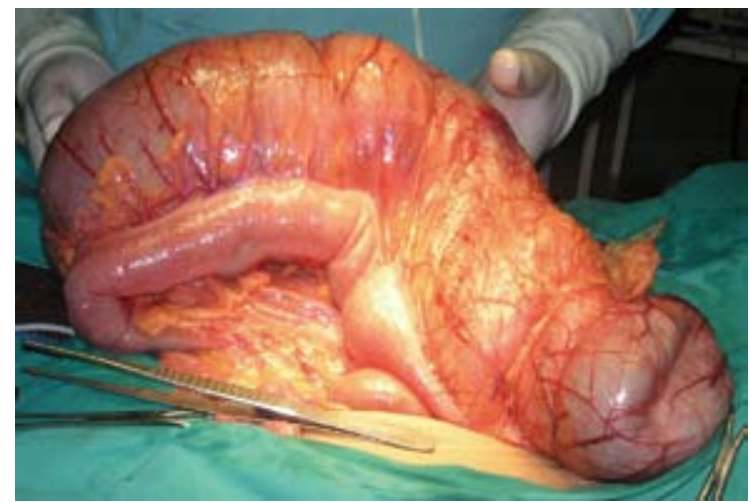

Figure 3 Operative view of normal transversal colon and the distended tubular colonic duplication.

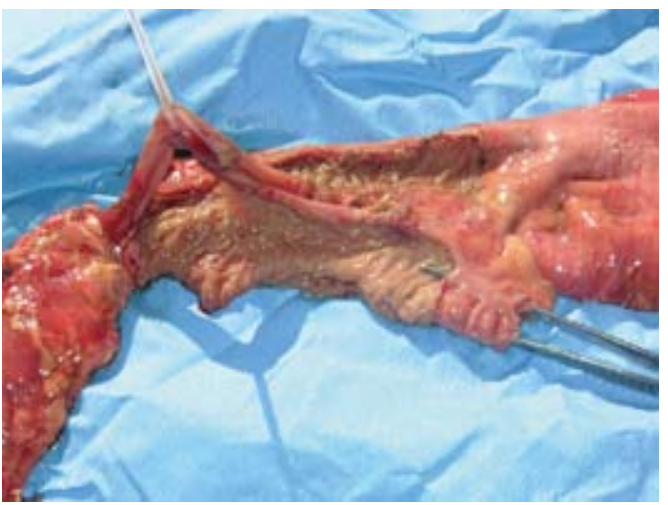

Figure 4 Surgical specimen showing part of the normal ascending and transversal colon together with a tubular duplication with all layers of the colonic wall dividing these structures.

Finally, right hemicolectomy was performed. The patient functioned well postoperatively and a few months after the procedure did not have any stool evacuation difficulties as encountered preoperatively.

\section{DISCUSSION}

Gastrointestinal duplications are rare congenital anomalies which in $80 \%$ of cases are detected prenatally or in the first two years of life, although they can remain undetected until older age $\mathrm{e}^{[6]}$. The clinical picture varies according to their location, size, and other factors such as the presence of ectopic mucosa within the duplication, communication with adjacent bowel, or inflammation. The duplications are classified according to their location (ileum 30\%, ileocecal valve $30 \%$, duodenum $10 \%$, stomach $8 \%$, jejunum $8 \%$, colon $7 \%$, rectum $5 \%$ ), shape: cystic $(\geqslant 80 \%)$ or tubular ${ }^{[7]}$. The most common clinical manifestations are abdominal pain and intestinal obstruction. Rarely, such duplications present with signs of acute abdomen or acute bleeding ${ }^{[8]}$. Pyloric and duodenal duplications can mimic hypertrophic pyloric stenosis or a choledochal cyst ${ }^{[5,9,10]}$. An ovarian cyst should be considered in female patients, and adolescent patients can sometimes have a presumptive diagnosis of Crohn's disease. Ultrasound is the imaging modality of choice for the evaluation of an abdominal mass in the neonate. Approximately $30 \%$ of the intestinal duplications 
are diagnosed prenatally ${ }^{[11]}$. Additional imaging procedures are often required: barium enema, CT scan, radionuclide scan (HIDA scan for choledochal cyst and pertechnetate scan for Meckel's diverticulum). However, gastric, duodenal, and rectal duplications can lead to a diagnostic dilemma.

Chronic constipation as a presenting symptom of intestinal duplication in adults present specific diagnostic problems because of a wide variety of etiologic factors. The cause of constipation is usually multifactorial. Secondary constipation could be caused by low fibre diet, anal fissures, thrombosed hemorrhoids, strictures, and tumors, hypercalcemia, hypokalemia, hypothyroidism, diabetes mellitus, pregnancy, stroke, Hirschsprung disease, Parkinson's disease, multiple sclerosis, spinal cord lesion, Chagas disease, familial dysautonomia, scleroderma, amyloidosis, mixed connective-tissue disease and use of different drugs. Functional constipation could be caused by repressed defecatory urge, irritable bowel syndrome, idiopathic megacolon or megarectum, idiopathic slow transit constipation, chronic intestinal obstruction, anismus, solitary rectal ulcer, intussusception, descending perineum, rectocele or ineffective straining.

Intestinal duplications often require urgent surgical intervention. Secretion of ectopic gastric mucosa causes ulceration of the intestinal mucosa, which can lead to massive bleeding or bowel perforation ${ }^{[12]}$. Gastric mucosa was found in $50.8 \%$ of cystic duplications and was present in all locations, including the colon and rectum with somewhat lower frequency of $33 \%{ }^{[3]}$.

Colorectal duplications represent $13 \%$ of all duplications ${ }^{[3]}$. Tubular duplications of the colon, may appear in a double-barreled or Y-shaped form ${ }^{[13,14]}$. These congenital anomalies have a double muscular layer and epithelium similar to the rest of the colon ${ }^{[13,15]}$. The clinical signs include constipation, vomiting, volvulus, perforation, and, most commonly, intestinal obstruction owing to the blind end of the duplication compressing the normal bowel $^{[13,14,16]}$. If there are no other associated malformations, tubular duplications of the colon are often not discovered until there is surgical treatment for the complications.

Most authors recommend that once the diagnosis is made, an elective surgical procedure should be performed to avoid complications and to perform the procedure in an optimal state of the patient, but few think that only symptomatic duplications should be surgically treated. The recommended surgical procedure is excision of the duplication. Intestinal duplications are located on the mesenteric side adjacent to normal bowel. Some duplications and the adjacent normal bowel share part of the muscular coat and the intestinal straight arteries. Thus, resection of both normal and duplicated bowels with intestinal anastomosis is a usual surgical treatment ${ }^{[17]}$. Sometimes it is possible to resect them without compromising the adjacent bowel vascularization. Although there have been some malignant changes reported in adults ${ }^{[18]}$, colorectal duplications are in themselves benign lesions. For this reason, surgical excision should not be radical but should involve complete resection of the duplication along with the relevant part of the colon ${ }^{[4]}$. Although rare, colorectal duplications are one possible cause of chronic constipation and, when discovered, should be treated surgically before complications arise. According to previous studies, surgical resection should extend at least $2 \mathrm{~cm}$ from the opening of the duplication; because substantial fibrotic changes near the opening of the duplication have been found ${ }^{[4,13]}$. Prognosis is good because of localized disease and the benign nature of the disease ${ }^{[4]}$.

\section{REFERENCES}

1 Ladd WE. Duplications of the alimentary tract. South Med J 1937; 30: 363-371

2 Macpherson RI. Gastrointestinal tract duplications: clinical, pathologic, etiologic, and radiologic considerations. Radiographics 1993; 13: 1063-1080

3 Puligandla PS, Nguyen LT, St-Vil D, Flageole H, Bensoussan AL, Nguyen VH, Laberge JM. Gastrointestinal duplications. J Pediatr Surg 2003; 38: 740-744

4 Holcomb GW 3rd, Gheissari A, O'Neill JA Jr, Shorter NA, Bishop HC. Surgical management of alimentary tract duplications. Ann Surg 1989; 209: 167-174

5 Cavar S, Bogovic M, Luetic T, Antabak A, Batinica S. Intestinal duplications--experience in 6 cases. Eur Surg Res 2006; 38: 329-332

6 Anderson MC, Silberman WW, Shields TW. Duplications of the alimentary tract in the adult. Arch Surg 1962; 85: 94-108

7 Stern LE, Warner BW. Gastrointestinal duplications. Semin Pediatr Surg 2000; 9: 135-140

8 Fotiadis C, Genetzakis M, Papandreou I, Misiakos EP, Agapitos E, Zografos GC. Colonic duplication in adults: report of two cases presenting with rectal bleeding. World J Gastroenterol 2005; 11: 5072-5074

9 Merrot T, Anastasescu R, Pankevych T, Tercier S, Garcia S, Alessandrini P, Guys JM. Duodenal duplications. Clinical characteristics, embryological hypotheses, histological findings, treatment. Eur J Pediatr Surg 2006; 16: 18-23

10 Lavine JE, Harrison M, Heyman MB. Gastrointestinal duplications causing relapsing pancreatitis in children. Gastroenterology 1989; 97: 1556-1558

11 Foley PT, Sithasanan N, McEwing R, Lipsett J, Ford WD, Furness M. Enteric duplications presenting as antenatally detected abdominal cysts: is delayed resection appropriate? J Pediatr Surg 2003; 38: 1810-1813

12 Wardell S, Vidican DE. Ileal duplication cyst causing massive bleeding in a child. J Clin Gastroenterol 1990; 12: 681-684

13 Correia-Pinto J, Romero R, Carvalho JL, Silva G, Guimaraes H, Esteveo-Costa J. Neonatal perforation of a Y-shaped sigmoid duplication. J Pediatr Surg 2001; 36: 1422-1424

14 Espalieu P, Balique JG, Cuilleret J. Tubular colonic duplications. A case report and literature review. Anat Clin 1985; 7: 125-130

15 Shin KS, Lee NH, Kim SY. An unusual case of colonic duplication causing chronic constipation in a child. J Pediatr Surg 1999; 34: 1410-1412

16 Ryckman FC, Glenn JD, Moazam F. Spontaneous perforation of a colonic duplication. Dis Colon Rectum 1983; 26: 287-289

17 Grosfeld JL, O'Neill JA Jr, Clatworthy HW Jr. Enteric duplications in infancy and childhood: an 18-year review. Ann Surg 1970; 172: 83-90

18 Heiberg ML, Marshall KG, Himal HS. Carcinoma arising in a duplicated colon. Case report and review of literature. $\mathrm{Br} J$ Surg 1973; 60: 981-982 